\title{
有机化学专业核心课程群教学质量提升改革实践
}

\author{
王云侠, 白银娟, 李剑利, 赵军龙, 张世平, 魏青, 王兰英 \\ 西北大学化学与材料科学学院, 国家级化学实验教学示范中心, 西安 710127
}

\begin{abstract}
摘要: 针对当前有机化学专业核心课程群教学中存在的问题, 开展了相应的教学质量提升改革。通过细化课堂教学实 施方案, 引导学生课前主动预习; 通过分段式课堂教学, 提高学生课堂参与度; 通过多层次的课后练习, 提高学生的 综合能力; 通过提高考题的高阶性, 引导学生注重思考能力和综合能力的发展。经过这样的教学质量提升改革, 学生 学习的主动性、思考能力和综合能力得到了显著的提高, 为培养知识、能力和思维全面协调发展的创新人才奠定了坚 实的基础。
\end{abstract}

关键词: 有机化学; 核心课程群; 教学改革; 学习主动性; 思考能力; 综合能力

中图分类号: G64; O6

\section{Reform Practice of Teaching Quality Improvement in Core Courses of Organic Chemistry}

\author{
Yunxia Wang, Yinjuan Bai, Jianli Li, Junlong Zhao, Shiping Zhang, Qing Wei, Lanying Wang * \\ National Demonstration Center for Experimental Chemistry Education, College of Chemistry \& Materials Science, Northwest \\ University, Xi'an 710127, P. R. China.
}

\begin{abstract}
The teaching quality improvement reform is carried out in this paper, in view of the problems existing in the teaching of the core courses of organic chemistry. Through refining the teaching implementation plan, the students are guided to preview actively before class. Through the segmented class teaching, students participate more efficiently in the class. Through the multi-level practice after class, the students' comprehensive ability is improved. By improving the challenge of the course test, students are guided to focus on the development of thinking skills and comprehensive capability. After a school year's teaching reform practice, the learning initiative, thinking ability and comprehensive ability of students have been significantly improved. This lays a solid foundation for the cultivation of innovative talents with knowledge, ability and comprehensive thinking.
\end{abstract}

Key Words: Organic chemistry; Core courses; Teaching reform; Learning initiative; Thinking ability; Comprehensive ability

课堂教学质量直接影响大学所培养人才的质量, 因此通过教学改革来提高课堂教学质量, 培养 知识、能力和思维全面协调发展的创新人才, 是教育界永恒的研究课题 ${ }^{[1-5]}$ 。有机化学专业是大学化 学学科重要的专业之一, 其中有机化学、波谱原理及解析和有机化学实验这三门课程构成了有机化 学专业的核心课程群。有机化学主要讲授有机化合物的结构、反应、反应机理和制备, 属于实验性 学科的理论课 ${ }^{[6,7]}$, 我们学院分配了 108 个学时; 波谱原理及解析主要讲授有机化合物紫外-可见吸

收稿：2019-06-21; 录用：2019-08-19; 网络发表：2019-08-29

“通讯作者, Email: wanglany@nwu.edu.cn

基金资助：陕西高等教育教学改革研究项目(17BY029); 教育部产学合作协同育人项目(201802314006); “陕西省一流专业建设” 项目; 西北大 学研究生质量提升工程项目(2019): 波谱分析; 西北大学教学研究与成果培育项目(JX14046, JX17076, JX17077, JX17079) 
收光谱、红外光谱、核磁共振谱和质谱等各类图谱的原理、特征及解析, 重在理解有机化合物的结 构与图谱之间的关系 ${ }^{[8]}$, 我们用 54 个学时讲解; 有机化学实验则是有机化学和波谱原理及解析这两 门课程的综合实践, 培养学生的有机化学实验技能和波谱分析能力, 注重对学生动手能力和实验综 合能力的训练 ${ }^{[9]}$, 我们用 144 个学时授课。其中, 有机化学和波谱原理及解析这两门课程是有机化 学实验对应的理论课程, 有机化学也是波谱原理及解析的基础。综上可见, 这三门课程既相对独立, 又互相紧密联系。因此, 如何从课堂着手, 引导学生学好有机化学和波谱原理及解析这两门理论课 程, 并能高效、灵活地将这些理论与有机化学实验相联系, 从而融会贯通并综合运用, 是有机化学 专业核心课程群教学的一个重要研究方向 ${ }^{[10,11]}$ 。

近年来我校有机化学专业核心课程团队教师在夯实专业基础的同时, 积累了丰富的教学经验, 教学质量已取得了长足进步 ${ }^{[12,13]}$ 。但目前依然存在一些问题, 归纳起来大致有以下三类: (1) 学生学 习主动性不够, 对教师依赖过多。这体现在部分学生没有课前预习及课后复习的习惯, 遇到问题不 善于运用所学知识和方法提出自己的想法, 而是直接抛给教师; 希望教师课堂上 “满堂灌” , 多讲 知识点, 课后多辅导讲解。(2) 学生对知识掌握的不够深透。例如有机化学课上讲完一个有机反应和 机理后, 接下来照猫画虎的例子学生可能会, 但是遇到一个需要结合机理才能推测出来的例子学生 可能就不会了; 对于波谱原理及解析中官能团的特征比较清楚, 但是遇到一系列化合物中特定官能 团波谱特征的变化趋势分析时就不会解释或推测。(3) 学生综合运用知识的能力较差。例如在乙酰 水杨酸制备实验中, 要求鉴定产物中是否存在原料水杨酸, 可学生想不到有机化学课程中讲到的苯 酚的定性反应。

仔细分析这三类问题, 其实就是由于学生学习主动性不够高, 死记硬背的多、思考的少, 最终 导致了综合能力不足的问题。伟大的科学家爱因斯坦曾经说过: “大学教育的价值, 不在于学习很多 事实, 而在于训练大脑会思考。” 可见思考在学习中扮演的角色尤为重要 ${ }^{[10]}$ 。因此结合我们教学中存 在的问题, 为了提高学生学习的主动性, 培养他们的思考能力和综合运用知识的能力, 我们对核心 课程群的教学进行了如下改革。

\section{1 细化课堂教学实施方案, 引导学生课前主动预习}

为了引导学生课前主动高效地预习, 教师首先结合教学大纲、课时和学生基础, 精心备课, 并 科学合理地将有机化学和波谱原理及解析这两门理论课的教学内容分为典型知识点和其他知识点两 部分。其中典型知识点是用于教师课堂讲解的教学内容; 其他知识点是用于学生课堂讲解的教学内 容。接着将学生分组, 并布置预习内容, 要求学生认真预习并与同学合作准备课堂讲解内容, 其中 预习中遇到的问题, 可以通过学生讨论或者与教师讨论的方式解决。针对每次课的教学内容, 我们 会有专门的课前小测验检查学生预习情况, 测验及格的学生认为预习是合格的。有机化学实验要求 学生从实验原理、实验所需试剂和仪器、具体操作、结果与讨论和注意事项等方面全程认真预习。 课前检查下列四项: 实验原理、所需试剂的物理常数和一般性质、所需仪器和具体操作步骤, 合格 的同学才能进入实验课堂。

比起传统的教学方式, 这种改革使得教师和学生课前的工作量均大幅增加, 因此我们选取人数 较少的基地班进行教学改革实践。其中有机化学选择典型反应, 例如卤代烃的亲核取代反应、烯烃 的亲电加成反应、芳烃的亲电取代反应、醛酮的亲核加成反应和羧酸衍生物酰基碳的亲核取代反应 等进行分解教学, 其中教师详细讲解每类反应中的两到三个典型反应, 其他反应让学生进行课堂讲 解; 波谱原理及解析选取红外光谱和核磁共振氢谱的影响因素及特征进行分解教学, 其中教师讲解 这两种图谱的影响因素, 学生则讲解各类化合物的红外和核磁共振氢谱特征部分。结果发现, 大部 分学生都能跟上节奏, 认真地预习布置内容。学生课后反映, 带着任务的预习比之前宽泛的预习对 于他们来说责任更大、任务更多, 但这种方式督促了他们主动学习和思考, 把预习落到了实处, 学 习效果也明显提高。 


\section{2 分段式课堂教学, 提高学生课堂参与度}

\section{1 理论课分为教师精讲、学生讲解和讨论总结三个阶段}

有机化学以结构决定化学性质, 机理决定反应特点这两条主线分别讲解不同官能团类化合物的 反应以及特点。在介绍每章内容时教师引导学生分析每类化合物的结构, 预测化学性质, 并精讲少 量典型反应, 其余的反应让学生在预习和听教师讲解的基础上自行讲解, 最后教师与学生讨论并对 课堂教学进行点评和总结, 从而全面掌握每类化合物的化学性质。例如烯烃的反应这部分内容, 教 师分析烯烃结构的特点, 引出烯烃的主要反应类型: 亲电加成、还原和氧化等。其中亲电加成部分, 教师以烯烃与卤化氢和卤素的亲电加成反应为例, 讲解该类反应的机理、区域选择性和立体选择性 等特点。接下来烯烃与水、醇、氢㲵酸和次卤酸等的反应就让学生试着讲解。波谱原理及解析以有 机化合物结构和波谱特征的关系为主线, 教师讲解波谱测定原理及基本影响因素, 让学生在此基础 上对各类化合物的波谱特征进行具体讲解, 最后教师对课堂教学进行总结。例如核磁共振氢谱这部 分内容, 教师讲解基本原理、谱图的组成, 以及氢化学位移的影响因素, 接下来学生对具体化合物 的氢谱特征进行讲解, 并进行比较分析。

改革实践发现, 教师讲解典型知识点时, 运用启发式教学法、典型案例教学法、类比式教学法 和引导式教学法等, 可以充分激发学生的学习兴趣。例如在讲到对映异构体这章内容时, 教师引入 “反应停” 这一手性药物, 并抛出海豹贞儿事件。这时学生明显地都在认真听讲, 并积极地思考其 中的原因, 接着教师再告诉他们原因, 就会让学生深刻意识到一些对映异构体在手性环境中性质差 异是很大的。这种典型案例教学法的运用, 活跃了课堂气氛, 也明显提高了教学效果。

学生讲解环节, 课前预习充分的学生, 在课堂上跃跃欲试, 积极展示自己准备的讲解内容, 课 堂气氛较前大幅度活跃。其中有的学生用板书、有的用课件、有的两者结合, 细心的同学还准备了 教学模型辅助讲解。我们每次请不同的学生进行讲解, 这样教师可以很好地了解学生以及小组的预 习情况, 也有利于同学之间互相学习。

师生讨论总结环节, 教师首先肯定学生讲解恰当或者进步的地方, 接着对学生讲解进行必要的 完善和补充, 对整个课堂内容进行总结。

这样的课堂教学改革, 使课程进度要比传统教学慢。为此, 我们采取了一些办法掌控课堂节奏 和教学进度。首先, 我们对传统课堂的教学知识点进行篎选, 重要知识点选择在课堂上优先讲解, 其余简单的知识点让学生课后自学; 其次, 落实预习环节, 加强了预习环节的管理, 检查学生课前 的预习情况, 预习通过才能上课, 否则不能上课。这两种方法实施后, 我们就可以顺利地完成了大 纲规定的教学任务。值得欣慰的是, 分段式课堂教学改革后, 课堂上随时提问以及课后主动提问的 学生人数都明显增加。对学生的问题我们不是直接给出答案, 而是先引导学生思考, 提示、讨论后 才会给出解答, 重在引导学生学会思考、掌握学习方法, 而不仅仅是知道问题的答案。这种课堂教 学改革也使得教师每次课都能看到学生的独特表现, 感受到学生在不断进步; 同时教师根据学生的 表现和反馈, 也不断地更新完善教学方法, 教学互动更加充分。

\section{2 有机化学实验课包括教师讲解、实验开展和讨论总结三个阶段。}

学生在预习合格的前提下, 进入实验课堂, 教师对实验过程进行简单讲解, 接着教师全程指导, 学生独立开展实验, 最后对整个实验进行讨论总结。学生实验过程中出现的问题, 教师要及时指出 来, 并引导学生思考解决。与理论课相比较, 实验课的讨论总结部分要占用比较多的时间, 旨在要 求学生不仅完成实验, 更要结合理论知识对实验过程和结果透彻理解。实验课强调 “不以成败论英 雄” , 实验操作规范、结果好的给予肯定; 操作不规范或者结果不好, 但是善于思考找原因, 并分 析合理, 我们也给予同样充分的肯定。学生尤其对课后的集中讨论感兴趣, 因为学生和老师可以充 分讨论交流。学生反映这种教学方式不仅锻炼了他们的动手能力, 更为他们提供了交流、讨论和落 实课堂效果的机会, 从而提高了他们的综合实验能力和思维能力。 


\section{3 多层次的课后练习, 提高学生的综合能力}

课后基础类和简单应用类的练习是学生必须完成的, 在此基础上, 我们提供综合应用和思考拓 展类的问题供学生自主拓展练习, 旨在向学生逐步展示基础知识的综合应用以及学科发展的最新成 果, 从而全方位提升学生的思考能力和综合能力, 并拓展学生的科研视野。例如有机化学课程中学 习了苯酚与三氯化铁生成蓝紫色络合物的反应, 可以用来鉴定苯酚。我们课后就给学生布置了思考 题: 这类显色反应中羟基的特征是什么? 醛酮羰基的烯醇式是否也可以发生该显色反应? 阿司匹林 (乙酰水杨酸)的制备实验中, 怎样鉴定产物中是否还有原料水杨酸? 看到这些问题, 学生们就进一 步去查阅参考书, 最终了解凡具有烯醇结构的化合物可以发生该显色反应, 因此醛酮的烯醇式可以 发生该显色反应; 阿司匹林的制备实验中, 也可以用该显色反应鉴定产品是否还有水杨酸。再例如 学习酮式和烯醇式互变异构时, 让学生解释为什么乙酰乙酸乙酯可以与饱和亚硫酸氢钠、氢氰酸和 差胺等反应, 也可以与金属钠或溴的四氯化碳溶液反应; 也让他们解析乙酰乙酸乙酯的核磁共振氢 谱、碳谱; 同时解释乙酰乙酸乙酯在正己烷和乙醇两种溶剂中紫外光谱图不同的原因。通过以上化 学性质、核磁共振谱和紫外光谱分析, 学生会全面地了解乙酰乙酸乙酯通常是酮式和烯醇式共存的 平衡体系, 而且溶剂对该平衡也有影响。例如学习了醇的氧化反应, 让学生思考酒驾的检测原理, 将反应和实际应用联系起来, 激发学生的学习兴趣, 加深学生对反应的理解。例如学习了 Beckmann 重排反应, 我们会提供文献中非经典 Beckmann 重排的反应, 让学生结合课堂所讲机理, 推导出该 文献反应的机理, 从而对 Beckmann 重排反应进行巩固和拓展。学生反映通过这样多层次的课后练 习, 他们可以很容易地将有机化学核心课程群的理论和实验互相联系, 融会贯通, 从而深入地理解 并掌握课程内容, 同时锻炼了他们的思考能力和综合应用能力。

\section{4 提高考题的高阶性, 引导学生注重思考能力和综合能力的发展}

与传统考试内容不同, 我们在考试中尽量避免简单记忆性题目, 适当减少基础类知识点的直接 考查, 增加了分析思考类和综合应用的题目, 从而引导学生注重思考能力和综合能力的发展。考试 方式也由一学期的期中和期末两次考试改为分章节考试, 一学期五到六次考试, 考试选择在辅导课 时间进行, 避免考试影响课程进度, 最后再结合平时成绩综合评价, 其中平时成绩包括课前预习、 课堂表现、课后作业和实验报告等环节。例如有机化学考题分为完成反应、简答题目、机理题目和 合成设计等类型; 波谱考题包括简答题和推结构等类型; 有机化学实验则是完成一个完整的合成和 表征实验, 并回答相应的思考题。结果一学年下来, 从作业、实验报告、卷面情况和课程论文等可 以看出, 学生对知识点的分析思考和综合应用能力在逐渐提高。但也有个别学生表现一直较差, 调 查发现他们课后复习巩固没做到位, 因此后面我们加强了课后复习巩固环节的检查工作, 使课堂教 学这个主体，在课前预习和课后复习的积极配合下稳步前进。

\section{5 结语}

总之, 有机化学核心课程群教学改革从课堂教学着手, 引导学生课前主动预习, 上课充分参与, 课后注重落实并进一步思考拓展, 从而显著地提高了学生的自主学习能力、思考能力和综合能力。 “路漫漫其修远兮, 吾将上下而求索”, 后面我们将继续完善和优化我们的教学质量提升改革, 为 培养知识、能力和思维全面协调发展的创新人才打下坚实的基础。

\section{参 考 文 献}

[1] 李志义. 中国大学教学, 2018, No. 12, 24.

[2] 辛爽, 潘玲, 刘群. 大学化学, 2018, 33 (4), 27.

[3] 于青, 王忠卫, 刘蕾, 黄小文. 大学化学, 2017, 32 (11), 12. 
[4] 韩杰. 化学教育, 2016, 37 (16), 24.

[5] 李厚金, 陈六平. 大学化学, 2018, 33 (10), 13.

[6] 邢其毅, 裴伟伟, 徐瑞秋, 裴坚. 基础有机化学. 第4版. 北京: 北京大学出版社, 2016.

[7] 李艳梅, 赵圣印, 王兰英. 有机化学. 第2版. 北京: 科学出版社, 2010.

[8] 常建华, 董绮功. 波谱原理及解析. 第3版. 北京: 科学出版社, 2017.

[9] 魏青. 基础化学实验(II) (有机化学实验). 北京: 科学出版社, 2011.

[10] 钱颖一. 清华大学教育研究, 2018, 39 (4), 1.

[11] 马䒯, 张恒, 苑世领, 胡清萍, 宋其圣. 高等理科教育, 2019, No. 2, 88 .

[12] 杨婕, 张世平, 孙伟, 白银娟. 大学化学, 2019, 34 (1), 82 .

[13] 王云侠, 赵宇, 王兰英, 李剑利. 大学化学, 2018, 33 (1), 69. 